\title{
Solving resource allocation problems in cognitive radio networks: a survey
}

\author{
Babatunde S. Awoyemi ${ }^{1 *}$, Bodhaswar T. J. Maharaj ${ }^{1}$ and Attahiru S. Alfa1,2
}

\begin{abstract}
Cognitive radio networks (CRN), in their quest to become the preferred next-generation wireless communication paradigm, will depend heavily on their ability to efficiently manage the limited resources at their disposal in meeting the demands of their numerous users and driving their operations. As a result, a considerable amount of research work has been recently dedicated to investigating and developing resource allocation (RA) models that capture the essentials of CRN. The various ideas put forward by researchers to address RA problems in CRN have been somewhat diverse, and somehow, there seem to be no links that bring cohesion and clarity of purpose and ideas. To address this problem and bridge the gap, in this paper, a comprehensive study on the prevalent techniques developed for addressing RA problems in CRN is carried out, with an intent to put some structure, relevance and meaning to the various solution approaches. The solution models are therefore grouped and/or classified based on certain outstanding criteria, and their strengths and weaknesses highlighted. Open-ended problems are identified, and suggestions for improving solution models are given. The study therefore gives good directions for further investigations on developing RA solutions in CRN.
\end{abstract}

Keywords: Cognitive radio networks, Resource allocation, Optimisation

\section{Review}

\subsection{Introduction}

The cognitive radio network (CRN) is no longer an entirely new concept in the wireless communication space. Since it started gaining attention over a decade ago, a plethora of technical materials in form of books, chapters in books, scholarly articles etc. have already been published on CRN. Consequently, a meaningless repetition of details about its ideas and ideals in this paper would be simply unnecessary. References [1-6] have all given clear and detailed explanations on the fundamentals of CRN.

Just to present a brief overview, the essential advances that formed the basis for the current surge in interest and drive in the development of CRN can be summarised as follows: demand for wireless communication has grown sporadically, and it is not likely to reduce any time soon; due to the increasing demand, there is a supposed

\footnotetext{
*Correspondence: awoyemibabatunde@gmail.com

${ }^{1}$ Department of Electrical, Electronic and Computer Engineering, University of Pretoria, Pretoria, South Africa

Full list of author information is available at the end of the article
}

spectrum scarcity problem, making it difficult to accommodate the rising wireless communication expectations; investigations reveal that the problem is, in fact, not that of an insufficient spectrum, but rather, of poor/inefficient utilisation of the already allotted spectrum by networks currently occupying them; an important solution to this problem has been provided in the form of dynamic spectrum access (DSA), whereby a spectrum will henceforth be dynamically allocated, and co-use and/or re-use of a spectrum space by more than one owner becomes a possibility; CRN emerged as the most potent driving force for the realisation of this new DSA paradigm.

In essence therefore, CRN, by depending on DSA, will be capable of delivering new and improved ways of managing the spectrum. DSA centres on sharing spectrum between licensed owners or primary users (PUs) and unlicensed owners or secondary users (SUs) of the spectrum. In earlier descriptions of DSA and CRN, SUs were designed such that they must be able to detect free spectrum spaces or holes, configure themselves to transmit in those frequencies, detect the return of PUs and immediately cease transmitting in those spectrum frequencies. Then, they must look for another free spectrum space,

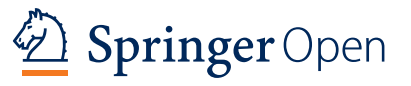

(C) 2016 Awoyemi et al. Open Access This article is distributed under the terms of the Creative Commons Attribution 4.0 International License (http://creativecommons.org/licenses/by/4.0/), which permits unrestricted use, distribution, and reproduction in any medium, provided you give appropriate credit to the original author(s) and the source, provide a link to the Creative Commons license, and indicate if changes were made. 
reconfigure themselves and resume transmission and be ready to vacate again should a PU return, all of these happening as seamlessly as possible. In more recent considerations of DSA and CRN, SUs may be enabled to transmit alongside PUs at the same time too, depending on the agreement between them. Usually when that is the case, the SUs transmit at low power over a wide bandwidth (e.g. ultra wide band) to minimise possible interference to the PUs. Further developments have however revealed that CRN are far and above just the ability to better manage or administer a spectrum. In her small but rich book on the essentials of CRN, Doyle conceptually described CRN thus: "the CRN must be a self-organising system it understands the context it finds itself in and can configure itself in response to a given set of requirements in an autonomous fashion. The configuration won't be on frequency or dynamic spectrum alone but on other features too like power, beam pattern, routing algorithm, coding techniques, filtering techniques etc. From the user point of view, the CRN will offer the benefit of personalising users' experiences so as to provide services tailored to the specific needs of individual users" [2]. It is therefore safe to say that, if these ideals of CRN, as predicted and promised, are eventually realised, the usefulness of CRN can be far and wide, and applications may cover a wide domain including areas such as the military, public safety, academia, health and commercial. The enormous promises of CRN therefore make it a technology on which several aspects of human communication life would have to rely heavily upon, and thus, it is such an important field to study and develop.

In terms of architecture, the most common architectural categorisation described in the literature classifies CRN as either centralised (infrastructure based), distributed (ad hoc based) or mesh architectures [7-10]. Another important description of the primary-secondary network architecture in CRN is based on the interference agreements between the two networks. This network architecture is described by the terms underlay [11], overlay [12, 13] and hybrid [14] networks. A third architecture commonly associated with CRN is cooperative and non-cooperative architectures. For the cooperative design, the SUs work together to make decisions on such things as spectrum sensing, so that their decisions are usually multilateral and centrally controlled $[15,16]$. Furthermore, cooperation can be between the PU network and the SU network $[17,18]$. Having established the above premises on CRN, the major interest in this paper is a look into the important aspect of resource availability for CRN, and to study the various methods that have been developed to fairly share and expeditiously utilise the limited resources at its disposal, in order bring it to the position of achieving its objective of becoming the preferred prototype for nextgeneration wireless communication. The remaining parts of this paper is organised as follows: Section 1.2 gives a general idea on resources in wireless communication, and the peculiarities that make such resources particularly scarce and/or limited for CRN; Section 1.3 provides a general description of resource problem formulations in CRN; Section 1.4 discusses the various approaches that have been developed and employed by researchers and authors in investigating solutions for their resource utilisation problems in CRN; observations on the solution models, as well as open-ended problems are discussed in Section 1.5; and finally, the concluding remarks are provided in Section 2.

\subsection{Resource allocation in cognitive radio networks}

Resources used up in wireless communication systems such as power, bandwidth and spectrum have always formed the backbone on which the operations of such systems depend. These resources being generally nonubiquitous, the various wireless communication models as developed have had to factor into their design the mechanisms by which their scarce resources are to be allocated or administered in order to achieve the most in their operations. Resource allocation (RA), which seeks to address that need, has therefore been an important aspect of all wireless communication networks. In fact, in several conventional wireless communication systems such as the orthogonal frequency division multiple access (OFDMA)based wireless networks, RA has been a rather active research topic. A few of the works that have addressed RA problems in OFDMA communication systems are found in references [19-25]. In general, RA problems in wireless communication essentially define how to optimise the limited resources in the communication network. RA problems are not therefore new and/or characteristic to the CRN. Particularly for the CRN, RA seek to jointly address the challenge of allocating its scarce resources, viz. spectrum allocation (frequency band, subchannels and time slots), power allocation, bit allocation, bandwidth, modulation schemes, data rates etc., in a manner that is fair to all users (primary and secondary) in the network.

There are generally two well-developed methods that have been actively adopted for addressing or describing RA problems in most wireless communication systems like the OFDMA-based networks. The methods are referred to as rate adaptive resource allocation (RARA) and margin adaptive resource allocation (MARA) models [22]. In RARA, the idea is usually to maximise a given function of the transmission rates, total capacity, fairness etc. of the subscribers or users under a total power constraint at the base station. Examples of RA problems developed and investigated as rate adaptive are found in the references [26-28]. Models that adopt the MARA method seek to minimise the total transmission 
power used up by the network while ensuring that the required transmission rates for all users are met. References [29-31] give examples of RA problems developed and investigated as margin adaptive problems.

Recent investigations have suggested that the methods developed for addressing RA problems in wireless communications (particularly the OFDM/OFDMA and its variants) are actually very adaptable to the RA problems in CRN as well [32]. It means therefore that, in generality, RA problems in CRN can similarly be broadly classified as either RARA or MARA. However, it is important to note that RA problems in CRN do pose a much higher level of challenge or difficulty than in other conventional wireless networks for several reasons. One important reason is the possible fluctuations in the available spectrum and hence the frequency and bandwidth of operation in CRN [33]. Another critical reason is the difficulty associated with, and the limiting effects of, considering CRN as an heterogeneous network. The heterogeneity of CRN would imply that in the design of CRN, the wireless network communication infrastructure must be capable of servicing a heterogeneous, probably incompatible, set of wireless consumer devices [34]. One other crucial factor that makes RA problems in CRN very challenging is the limitation in networking and productivity of CRN due to the level of permissible interference to either the PUs, or even among the SUs themselves. The limitation in SUs' transmission due to the level of permissible interference to PUs is probably the most crippling constraints to achieving great resourcefulness and optimal utility in CRN. The above reasons make it imperative to carry out detailed investigations on the basis and principles for adopting/adapting the developed methods for RA in other wireless communications to CRN. Such investigations will not only ascertain their suitability of application or purpose, but will also help to describe and analyse its workability. A considerable amount of work has already been carried out in this regard, as this literature study reveals, but also a lot more work is still required in order to bridge the research gaps.

\subsection{Resource allocation problem formulation in CRN}

There is a sizeable amount of research work on RA problems in CRN already. The various investigations have shown that, in almost all cases, RA problems in CRN are fully demonstrated to be optimisation problems. The knowledge of optimisation is therefore crucial to the understanding of, and in developing solutions to, RA problems in CRN. In essence, optimisation can be explored and employed as a vital tool for solving RA problems in CRN. Optimisation in itself is a well-developed analytical tool for solving a host of problems and is therefore used broadly in different fields of science such as mathematics, operations research, business finances and economics and engineering. In optimisation, there is usually an objective to be achieved, either that of maximising or minimising an entity or a number of entities, and this is always captured in the objective function. Then, there are certain limiting constraints that must be taken into consideration while seeking to achieve the objective. In solving, the constraints cannot be violated; otherwise, the solutions to such problems, if ever obtained, become void. The final component of almost all optimisation problems are the decision variables. The variables are the parameters to be obtained while solving, in order to arrive at (optimal) solutions. Due to space limitations and also to help keep focus on the subject matter, the preliminaries on optimisation are not discussed in this paper. Readers who are interested in gaining some fundamental knowledge on optimisation are directed to the following references for the needed help [35-38]. A general form of RA optimisation problem formulation in CRN is next provided.

The general formulation developed gives a description of what the objective functions usually are, as well as the constraints and the decision variables, and the interplay between them. Let $\mathbf{p}$ and $\mathbf{q}$ be two vectors of dimensions $a$ and $b$, respectively. Also let the set of positive integers $I=$ $\{0,1,2, \ldots\}$. Assume we need to obtain the values of $\mathbf{p}$ and $\mathbf{q}$ for which a function $f(\mathbf{p}, \mathbf{q})$ is maximum, given that there are a set of constraints $g_{i}(\mathbf{p}, \mathbf{q}) \leq n_{i}, i=1,2, \ldots, r$, and that each variable is non-negative. The above formulation can be written mathematically as:

$$
\max z=f(\mathbf{p}, \mathbf{q})
$$

subject to

$$
\begin{aligned}
& g_{i}(\mathbf{p}, \mathbf{q}) \leq n_{i}, i=1,2, \ldots, r, \\
& p_{j} \geq 0, j=1,2, \ldots, a, \\
& q_{k} \in I, k=1,2, \ldots, b .
\end{aligned}
$$

Equation (2) is more simply written as:

$$
\mathbf{g}(\mathbf{p}, \mathbf{q}) \leq \mathbf{n},
$$

where

$$
\mathbf{g}(\mathbf{p}, \mathbf{q})=\left[\begin{array}{c}
g_{1}(\mathbf{p}, \mathbf{q}) \\
g_{2}(\mathbf{p}, \mathbf{q}) \\
\vdots \\
g_{r}(\mathbf{p}, \mathbf{q})
\end{array}\right],
$$

and $\mathbf{n}=\left[n_{1}, n_{2}, \ldots, n_{r}\right]^{T}$. If the problem was a minimisation problem, the function $z=f(\mathbf{p}, \mathbf{q})$ could be easily transformed to a form of maximisation function by simply negating the objective function, i.e., $\max w=-f(\mathbf{p}, \mathbf{q})$. From the general formulation given above, Eq. (1) is the 
objective function, Eqs. (2)-(4) are the constraints, while $p_{j}$ and $q_{k}$ are the decision variables. As an example, Eq. (1) could be a maximisation of the total network capacity, vector $\mathbf{p}$ could be a set of transmission power for users, vector $\mathbf{q}$ could be subcarrier allocation, which would usually take integer values of 0 or 1 , and Eq. (2) could be the interference limit constraint or the power constraint.

Table 1 presents some examples of works/related works on RA problems in CRN that have either been addressed or sought to be addressed by various authors. The table also highlights the objective function, constraints and decision variables employed by these authors in achieving or seeking to achieve their goal. Although it is by no way exhaustive, the intend is to provide an idea on the different formulations developed by authors in achieving optimal RA in CRN.

From the general formulation of RA in CRN provided in Eqs. (1)-(4) (which represents succinctly most formulations on RA for CRN in the literature), it can be observed that the RA problems in CRN are best described as complex, non-deterministic polynomial-time hard (NP-hard) optimisation problems. NP-hard problems are problems which may be solvable in polynomial time, but then, only by a non-deterministic algorithm. The description of NP-hard problems are well-established, and references $[39,40]$ provide a good summary. The main point is that, obtaining solutions to NP-hard optimisation problems, into which category RA problems in CRN fall, though possible, can be very difficult and time consuming. Unfortunately, solutions to wireless communication problems, especially CRN, has to be timeous for it to be meaningful and useful. If the solutions take too long to be reached, premises and prevailing conditions on which the original problems were designed may have changed considerably, thus rendering the purported solutions unusable. One of the major issues, still open-ended, is on developing generalised RA models in CRN with reduced computational complexities, and that can provide timeous solutions. Finding meaningful and useful (applicable) methods for arriving at solutions to RA problems in CRN is therefore an exciting research focus. A review of the approaches developed by most researchers in the field is carried out in this paper.

\subsection{Classification of resource allocation solutions in CRN}

There are a number of approaches developed for solving the complex NP-hard RA problems in CRN that have been proposed and promulgated. In this section, the solution approaches are classified and critically examined. The basic features of each approach are highlighted, and so also the drawbacks. For clarification and ease of reference, the various approaches to solving RA problems in CRN can be classified into these broad perspectives:
- Obtaining solutions through classical optimisation;

- Obtaining solutions by a careful study of problem structure;

- Obtaining solutions by the use of heuristics or meta-heuristics (global optimisation);

- Obtaining solutions by applying game theory (multi-objective optimisation);

- Obtaining solutions through soft computing-based optimisation.

These categories are further discussed.

\subsubsection{Solutions using classical optimisation}

RA problems in CRN that fall into any of the welldeveloped classical optimisation methods can be solved optimally using the class of optimisation into which they fall. For instance, if a developed RA problem happens to be a linear programming (LP) problem, several established methods for solving such problems exist. Examples of methods for solving LP problems are simplex and interior point methods. In [41], the authors developed their frequency-time allocation problem in cognitive radio wireless mesh network as an LP problem and then employed the simplex method to obtain optimal solutions. In [42], the problem of optimally allocating PU bands to SUs was addressed and the optimisation problem used to obtain the stability region's envelope was shown to be, and solved as, an LP. Interior point method was used in [43] to address the problem of joint transmit beamforming and power control of SUs when they are allowed to transmit simultaneously with PUs. Furthermore, even when a RA problem is non-linear but if its convexity can be established, there are several known methods for solving convex optimisation problems that can be employed to solve such problems. One example of a method for solving convex optimisation problems is by using the Lagrangian duality method, usually with the application of the Karush-Kuhn-Tucker (KKT) conditions [44].

With the classical optimisation approach therefore, well-established tools are used in obtaining optimal solutions to the developed RA problems for CRN when such problems nicely fit into well-known optimisation structures. Other classical optimisation tools used for solving well-defined linear or convex programming RA problems are mostly off-shots of either the simplex or the interior point methods. Some of the most common methods and the corresponding references where they have been employed in obtaining solutions to RA problems in CRN are as follows: branch-and-bound (BnB) $[19,45]$, branchand-cut (BnC) [46], lift-and-shift ( $\mathrm{LnS})$ [47], iterative and double-loop iterative methods [21,23], dual decomposition $[21,48]$, Lagrangian duality $[48,49]$, barrier method $[50,51]$, gradient decent approach [52], column generation $[53,54]$ etc. In general, these methods, because of 
Table 1 Description of RA problem formulations in CRN

\begin{tabular}{|c|c|c|c|c|}
\hline References & Problem definition & $\begin{array}{l}\text { Objective } \\
\text { function }\end{array}$ & Main constraints & $\begin{array}{l}\text { Decision } \\
\text { variables }\end{array}$ \\
\hline$[55,70,71,90]$ & $\begin{array}{l}\text { Optimal RA in } \\
\text { MIMO-based CRN. }\end{array}$ & $\begin{array}{l}\text { Maximising the } \\
\text { achievable data } \\
\text { rate (or total } \\
\text { capacity) of SUs. }\end{array}$ & $\begin{array}{l}\text { Transmit power } \\
\text { limit of SUs, } \\
\text { interference } \\
\text { limit to PUs, total } \\
\text { transmission time } \\
\text { of SUs must be } \\
\text { equal to the time } \\
\text { slot duration. }\end{array}$ & $\begin{array}{l}\text { Number of SUs } \\
\text { served. }\end{array}$ \\
\hline$[50,91,92]$ & $\begin{array}{l}\text { Efficient RA } \\
\text { for CRN with } \\
\text { cooperation. }\end{array}$ & $\begin{array}{l}\text { Maximising the } \\
\text { sum rate of all } \\
\text { SUs. }\end{array}$ & $\begin{array}{l}\text { Transmission } \\
\text { power budget } \\
\text { of the SUs } \\
\text { and the relays, } \\
\text { interference to } \\
\text { PUs within its } \\
\text { tolerable } \\
\text { threshold, } \\
\text { each subchannel } \\
\text { can only be } \\
\text { allocated to } \\
\text { one SU. }\end{array}$ & $\begin{array}{l}\text { Achievable } \\
\text { rate over a } \\
\text { subchannel, } \\
\text { power allocated } \\
\text { to each } \\
\text { subchannel, } \\
\text { integer variables } \\
\text { of time slot and a } \\
\text { binary allocation } \\
\text { indicator }\end{array}$ \\
\hline$[49,83,84]$ & $\begin{array}{l}\text { Energy-efficient } \\
\text { RA for CRN with } \\
\text { imperfect sensing } \\
\text { and/or femtocells. }\end{array}$ & $\begin{array}{l}\text { Maximising } \\
\text { bandwidth } \\
\text { capacity for SUs. }\end{array}$ & $\begin{array}{l}\text { Power contraint } \\
\text { on SUs network, } \\
\text { minimum rate } \\
\text { guarantee for } \\
\text { some SUs and } \\
\text { best effort service } \\
\text { for the remaining } \\
\text { SUs, each } \\
\text { subchannel can } \\
\text { only be allocated } \\
\text { to one SU. }\end{array}$ & $\begin{array}{l}\text { Transmit power } \\
\text { for each SU, the } \\
\text { binary channel } \\
\text { allocation } \\
\text { indicator. }\end{array}$ \\
\hline$[45,61-63]$ & $\begin{array}{l}\text { Optimal RA in } \\
\text { MIMO-OFDMA } \\
\text { based CRN. }\end{array}$ & $\begin{array}{l}\text { Maximising sum } \\
\text { throughput of } \\
\text { the SUs. }\end{array}$ & $\begin{array}{l}\text { Interference } \\
\text { leakage to PUs } \\
\text { always below a } \\
\text { threshold, each } \\
\text { SU must achieve } \\
\text { the minimum } \\
\text { required data } \\
\text { rate, total } \\
\text { transmit power } \\
\text { of all SUs must be } \\
\text { below the } \\
\text { available power } \\
\text { at base station, } \\
\text { no more than } \\
\text { one SU is } \\
\text { allocated to each } \\
\text { subchannel. }\end{array}$ & $\begin{array}{l}\text { Data rate on each } \\
\text { subchannel. }\end{array}$ \\
\hline [67] & $\begin{array}{l}\text { RA for CRN with } \\
\text { opportunistic } \\
\text { access }\end{array}$ & $\begin{array}{l}\text { Minimising the } \\
\text { symbol error } \\
\text { rate of the } \\
\text { SUs' network } \\
\text { transmission. }\end{array}$ & $\begin{array}{l}\text { Constraint on the } \\
\text { maximum } \\
\text { individual powers } \\
\text { of each SU, a } \\
\text { minimum } \\
\text { number of } \\
\text { symbols must be } \\
\text { sent within a time } \\
\text { frame, constraint } \\
\text { on the minimum } \\
\text { acceptable } \\
\text { throughput of } \\
\text { the network, } \\
\text { interference } \\
\text { power to PUs } \\
\text { must be below a } \\
\text { certain threshold. }\end{array}$ & $\begin{array}{l}\text { Total power } \\
\text { available to } \\
\text { the system, } \\
\text { transmitted } \\
\text { symbol time, }\end{array}$ \\
\hline
\end{tabular}


their advantage of obtaining optimal solutions, are highly significant. Their utmost importance is in the fact that solutions provided, because they are optimal, can act as bounds for the suboptimal solutions obtained by the use of other approaches or methods. The major disadvantages of this approach are that; firstly, most RA problems in CRN do not fit nicely into any standard optimisation model, and secondly, proving convexity of most non-linear programming problems can be herculean, if not impossible to achieve. Also, obtaining solutions with this approach usually require high complexities and computation time.

\subsubsection{Solutions by studying problem structure}

As earlier mentioned, most RA problems in CRN do not fit into any standard optimisation model, and as such, directly applying classical optimisation to obtain solutions, in most cases, is highly improbable. A number of other techniques have been exploited and employed in seeking solutions. One important technique is by carefully studying the structure of such problems to see if there are any special feature(s) that can be exploited to either make such problems easier to solve, or to fit them into some classical optimisation models. Usually, this approach will either give optimal or suboptimal solutions, depending on how close the restructured or reconstituted problems are to the original problem formulation. Some known approaches based on the study of problem structure are examined below.

Solution by separation or decomposition Certain RA problems can be split into two or more simpler problems without significantly affecting the overall import of such problems. This implies that, by a careful study of the problem structure, an original RA problem can be separated or decomposed into two or more simpler sub-problems and each solved individually, usually with a lot less difficulty. The solutions are later combined to give the exact (or close to exact) final response to the initial problem. There are several methods of decomposition that have been used in solving RA problems in $\mathrm{CRN}$, as found in the literature. One such decomposition method is the Dantzig-Wolfe decomposition, and there are others. Examples of RA problems in the CRN that have employed decomposition in arriving at solutions can be found in references [21, 55]. In [55], the authors obtained optimal solution to their RA problem by using a primal-dual decomposition method whereby the overall problem is decomposed into individual power allocation sub-problems and solved for every decision variable pair. Authors in [21] divided their RA problem (joint spectrum and power allocation for multband CRN) into two stages and used an iterative dual decomposition method to solve it. In [56], the authors developed a CRN duality technique that decomposed their utility maximisation problem into three sub-problems-optimising SINR assignment, power and interference temperature. Similarly, the work in [54] used a decomposition approach in jointly addressing the problem of spectrum sensing, channel assignment and power allocation in cellular CRN. The initial problem, which was a mixed integer non-linear programming (MINLP) problem, was decomposed into two sub-problems-optimal spectrum sensing, and optimal channel assignment and power allocation-without sacrificing optimality. The advantage of this solution technique is the possibility of realising optimal solutions with reduced computational complexity. The major bottlenecks are that, not all problems are decomposable, and some problems loose a significant part of their imports when attempted to be decomposed into smaller sub-problems.

Solution by linearisation In almost all RA problems in CRN, the original problems, as developed, are usually non-linear in nature. Either the objective function is not a linear function or one or more of the constraints is/are not linear. Once the linearity of either the objective function or any/some of the constraints cannot be established, the problem is definitely a non-linear optimisation problem. A useful method for obtaining solution to non-linear RA problems in CRN is by seeking to linearise the non-linear expressions/constraints of the problem. If/once this can be achieved, obtaining solutions to the linear optimisation counterpart of the problem through classical optimisation becomes straightforward. The linearised expressions may indeed be approximates of the original, but if the values obtained are close estimates or within certain acceptable limits or bounds, the solutions provided by the new problem can be useful and meaningful, even though suboptimal. Examples of RA problems in which linearisation has been employed as a useful tool to achieve solutions can be found in [20, 57, 58]. In [58], a combination of linearisation, relaxation and reformulation techniques were employed in solving their RA problem. For the linearisation part, a constraint, which was non-linear due to the combination of multiplication and division operations, was transformed into a linear form by the use of the logarithm function. The problem's equivalency was maintained due to the monotonicity property of the logarithm function. The major advantage of this technique is the ease with which LP problems are solved as compared to non-LP, once the linearisation can be achieved. The major challenge with the technique is that, certain functions or expressions which commonly appear either in the objective function or constraints of RA problems in CRN are really difficult to find equivalent linear expressions for. 
Solution by relaxation Some RA problems in CRN are complex and difficult, mainly because of an integer constraint. Indeed, many problems that deal with channel (or subchannel) allocation are binary in nature, whereby a channel is either allocated to a user (assigned the value 1 ) or it is not (assigned the value 0). These kinds of problems can be solved a lot more easily by relaxing the integer constraint, i.e., by allowing the decision variable to take any value between 0 and 1 , rather than imposing it as either 0 or 1 . By rounding up or down, approximate solutions to those problems can be easily obtained. An example of where relaxation has been used in obtaining solution to a RA problem is in [59]. The RA problem, as developed, was a MINLP problem but by relaxing the integer constraint, the problem became an LP and was then solved. The work in [58] also employed integer relaxation in developing its solution model. The major issue with the relaxation approach is that only suboptimal solutions can be obtained, and in some instances, the gap between solutions obtained after relaxation and the optimal can be significantly wide apart.

Solution by approximation An important method for obtaining useful solutions to RA optimisation problems in CRN is through approximation. Certain functions, appearing in the objective function or the constraints, could be all that render an almost-linear problem nonlinear or a should-be convex problem non-convex thereby making the entire problem difficult to solve. If an approximate substitute to such functions can be obtained, the entire problem could become linear or convex, and obtaining solutions can be a lot easier. The substitute to such functions must of necessity be a close approximation of the initial functions before this method can be meaningfully employed. Again, only a suboptimal solution to the original problem can be achieved, but should the approximate substitute of the functions be good enough, the suboptimal solutions can be very close to the optimal, and therefore extremely useful. Importantly, the complexities in computations, problem analyses and time to arrive at solutions can be significantly reduced due to the approximation of such functions. There are examples in the literature of the use of approximation in obtaining solution to RA problems in the OFDMA-based networks, as well as CRN. In [24] for instance, the authors, in order to maximise total network utility, approximated their best-effort user utility function as a piece-wise linear function and proposed an LP-based cluster allocation algorithm for solving. The major setbacks with this approach are that the approximate representation of the original function could contain a number of extra variables, leading to an increase in the decision variables of the entire problem, and solutions obtained through approximate substitutes are usually suboptimal rather than optimal.
Solution by reformulation Another important approach used extensively to obtain solutions to NP-hard RA optimisation problems in CRN is through reformulation. By a careful consideration of the structure of a RA problem, certain distinct properties of the problem, once identified, can be exploited in arriving at a reformulation or regeneration of the original problem, and that without loosing its imports or details. The new or reformulated problem is, in most cases, an easier version of the original problem, and such that classical optimisation tools may be employed in arriving at viable solutions. This method has been applied in a number of RA problems in CRN, examples are in references [22, 45, 56, 60-63]. In [56], the authors, in an attempt to solve the utility maximisation problem for spectrum sharing in CRN, due to its non-convexity and tight coupling between power and interference, had to reformulate. The reformulated problem was an optimisation problem involving spectral radius constraint sets, and optimal solutions were obtained by using a tuning-free geometrically fast convergent algorithm. Authors in [60] developed algorithms for decision making to optimise radio resource usage in heterogeneous cognitive wireless networks. An important part of the solution was in the reformulation of the heterogeneous base station selection problem to a minimum cost-flow problem, which was then solved as a directional graph with low computational complexity. The works in [45, 61-63] have all followed a similar pattern of reformulating RA problems which were originally non-linear, non-convex NP-hard problems into integer linear programming (ILP) problems, and then solving optimally using a BnB optimisation technique. The main advantage of a reformulation approach is that optimal solutions can be obtained to seemingly difficult problems, and usually with much less computational complexity, once the special structure has been found and exploited in achieving the reformulation. The sole drawback of this method is the difficulty in finding that special structure that can be exploited in certain RA problems.

\subsubsection{Solutions by heuristics or meta-heuristics}

A very popular approach, used in many occasions to obtain solutions to RA problems in CRN, is through the development of problem-specific heuristic(s). Certain problems will be almost certainly impossible to solve through classical optimisation, no matter what 'trick' is sought for or employed to try make the problem solvable. Even in situations where any of the already-discussed methods (such as linearisation or approximation) succeed and the RA problems become solvable, in most cases, it is still unlikely that such optimal or suboptimal solutions would be obtainable at a reasonably feasible time frame for practical purposes or real-life scenarios. The complexities of the problem would, in all probability, make 
the solutions impracticable, most especially for large networks. It therefore means that, methods for obtaining much faster solutions with less computational complexities must of necessity be devised. In most cases therefore, a heuristic is always developed alongside the solution provided through any of the aforementioned methods, so as to achieve this goal of producing solutions in a reasonable time frame that is good enough for solving the problem at hand.

In developing heuristics, logical reasoning and not necessarily analytical or numerical derivations on how to solve a particular problem is pursued. The solutions to the RA problems in CRN using heuristics are thus problem-specific, almost certainly non-transferable to solve other RA problems, and usually only provide suboptimal solutions. The advantages with heuristics are that problems which may not be solvable by classical optimisation may be solved by developing a heuristic for it and that such solutions are usually obtained at a much more reduced time frame, even with large networks. Examples of heuristics developed and employed in solving RA problems in CRN are given below:

- Greedy algorithms: In greedy algorithms, the heuristic is developed in such a way that it selects whatever is currently or immediately the best next step, regardless of whether or not there could be some better steps later. Variants of the greedy algorithm are selective greedy and distributed greedy algorithms. References [12, 19, 20, 64, 65] have all employed greedy algorithms in obtaining solutions to their RA problems in CRN. Solutions provided are not usually optimal but they can be obtained at a reasonably good time.

- Water-filling schemes: Several water-filling heuristics (and its variants) have been developed to solve RA problems in CRN. The water-filling schemes developed from the idea of the water jug problem. Examples where these schemes have been employed in solving RA problems in CRN can be found in references [66-71]. The methods are simple to develop and they give very close-to-optimal solutions with reduced complexities.

- Preassignment-and-reassignment algorithms: In preassignment, certain amount of resources, subchannel or power for instance, are initially pre-allocated as base resources to some or all users before the other resources are now optimally shared among the remaining users. As the algorithm runs, more resources are allocated to all or a category of users to achieve a higher overall capacity or productivity. After one or more runs, the algorithm may check that the constraints are not violated and should there still be some residual resources, a reallocation (or reassignment) of resources is again carried out to seek to improve the overall utility of the network. Examples of the use of this heuristic method can be found in references $[19,72]$.

- Recursive-based and/or iterative-based heuristics: These methods carry out allocation of resources either recursively or iteratively to all users in the network. While iteration uses a repetition structure, recursion uses a selection structure. Importantly, both methods steadily increase utility until further iteration or recursion results in a negligible amount of improvement, and thus, a termination is evoked. References [51, 61] have applied these techniques in developing their heuristics to solve their RA problems.

Meta-heuristics are developed for solving computationally demanding RA problems. They are generally wide-ranged and are more employed for problems that have the possibility of obtaining a number of local 'optimal' solutions, or such problems for which there is no satisfactory problem-specific algorithm to solve them. A meta-heuristic is thus an algorithm designed to solve approximately a wide range of hard optimisation problems, without having to deeply adapt to each problem [73]. Meta-heuristics involve using tricks so that the algorithm does not get stuck around a local minima or maxima, whereas a better optimal solution could still have been realised. Some examples of meta-heuristics that have been used for RA in CRN are given below:

- Genetic algorithms: Genetic algorithms are used by defining resources in the form of chromosomes and genes and the users' QoS requirements are given as input to the algorithm procedure. An example is found in reference [74] where genetic algorithm was used in optimising spectrum allocation in CRN. Genetic algorithm was also used in [75] for optimising spectrum utilisation while providing a fairness guarantee between users in CRN.

- Simulated annealing: In this technique, by the process of iterative controlled 'heating' and 'cooling' of the search space, an optimal 'temperature' is found which corresponds to an optimal utility. References $[76,77]$ have used this technique in solving allocation or utility maximisation problems in CRN.

- Evolutionary algorithms: These are algorithms that have some inclination towards simulating the evolution of individual structures via processes of selection, recombination and mutation reproduction, thereby producing better solutions. Examples are coco search, ant colony, particle swarm optimisation, 
bee colony etc. In [78], authors used the particle swarm optimisation in realising power allocation in CRN. Authors in [79] used the bee colony idea in achieving relay assignment with power control in CRN.

- Tabu searches: This algorithm explicitly uses the history of the search, both to escape from local minima and to implement an explorative strategy. The main characteristic of this approach is indeed based on the use of mechanisms inspired by the human memory. An example of its use in solving RA problems in CRN is found in [80] where the method was applied to achieve channel allocation optimisation for all users of the network.

From the explanations given above, it can be said that heuristics and meta-heuristics are indeed powerful tools for obtaining solutions, especially for large, practical networks. The major limitation with these methods are the deficiencies in analytical/numerical representations of the problems and the non-transferability of the knowledge acquired in solving a problem to help solve other problems.

\subsubsection{Solutions by multi-objective optimisation}

An important approach to solving RA problems in CRN, especially problems that are multi-objective in nature, is the use of game theory. Actually, some developed RA problems in CRN are multi-objective optimisation problems in that they require a process of simultaneously optimising two or more conflicting objectives, subject to certain constraints. One method that has been employed in addressing multi-objective optimisation problems is in converting them to single-objective optimisation problems by using techniques such as reducing dimension, Min-Max method, the ideal point method, the weighted sum of squares method, the virtual target method, sequencing method, feasible direction method, the centre method, interactive programming method etc. However, good as these techniques are, there are instances where conventional optimisation models may not be adequate in addressing such multi-objective problems, hence, the use of other multi-objective solution techniques such as game theory [81]. Several game models exist, and some of them have been employed in solving multi-objective RA problems in CRN. Some examples and the corresponding references where they have been applied are cooperative game [70, 71], non-cooperative game [82] Nash bargaining (Pareto optimisation) $[17,18]$ and Stackelberg game $[83,84]$.

\subsubsection{Solutions through soft computing}

A very new/recent approach to solving RA optimisation problems in CRN is through soft computing-based optimisation. In this approach, software/computer-based programming are used in allocating resources to users within the network. The developed programmes use intelligent techniques such as artificial intelligence, neural networks, Q-learning and fuzzy systems in driving the optimisation processes [85]. In [86] for instance, the authors used a special type of Q-learning, called multiagent reinforcement learning, in achieving RA for multiuser CRN. During the learning process, each SU sees the channel and other secondary users as its environment, updates its Q-values and takes the best action based on the prevalent situation. Authors in [87] used an artificial intelligence technique in developing a decision-making tool for allocating resource in CRN. In the developed model, cognitive radio learning inference and decision-making engine based on Bayesian network was proposed to obtain the optimum configuration rules to adapt to the variation of the environment with the learning and inference algorithm of Bayesian network. In [88], the authors proposed a fuzzy neural system for spectrum allocation in CRN. In the model, parameters such as spectrum utilisation efficiency, degree of mobility and distance to the PUs of CRN are given as inputs to the fuzzy logic decision-making process, while the output of that process gives the spectrum access decision, based on linguistic knowledge of some predetermined rules. The major challenge with this approach is that the soft computing techniques, such as artificial intelligence and neural networks, are very difficult and complex to develop, analyse and apply in real-life scenarios.

In summary, there is an ample number of methods that have been developed for solving RA problems in CRN and these methods are usually exploited by researchers in obtaining solutions to their formulated RA problems. In this study, the most critical ones have been grouped and their workability explained. Both the strong points of these methods, as well as their weak areas, have been highlighted. Table 2 contains the summary of the solution models discussed.

\subsection{Observations and open-ended problems in resource allocation for CRN}

The authors' important observations and/or opinions on these solution approaches/schemes are given below:

- Generally, there seems to be a kind of disjointedness in the RA problem development, as well as in solution models developed by the various authors. The objective functions for even seemingly similar problems are usually diverse, and so are the constraints and decision variables employed. It therefore seems difficult to find any form of coordination or focal point in the problem definitions. Similarly, the ideas put forth for investigating solutions lack any proper order or a particular standard. 
Table 2 Summary of solution approaches to RA problems in CRN

\begin{tabular}{|c|c|c|c|c|}
\hline $\mathrm{S} / \mathrm{N}$ & Solution approaches & $\begin{array}{l}\text { Solution methods and/or } \\
\text { models }\end{array}$ & Features & Drawbacks \\
\hline 1. & $\begin{array}{l}\text { Classical optimisation e.g. } \\
\text { LP, convex optimisation } \\
\text { etc. }\end{array}$ & $\begin{array}{l}\text { Simplex and its variants } \\
\text { (BnB, BnC, LnS, implicit } \\
\text { enumeration etc.); inte } \\
\text { rior point method and its } \\
\text { variants (barrier method, } \\
\text { Newton's method etc.); } \\
\text { Lagrangian duality; } \\
\text { knapsack; travelling } \\
\text { salesman problem etc. }\end{array}$ & $\begin{array}{l}\text { Approach gives optimal } \\
\text { solutions; solutions act as } \\
\text { bounds (upper or lower) to } \\
\text { other solution models. }\end{array}$ & $\begin{array}{l}\text { Usually, most RA problems } \\
\text { do not fit into any class } \\
\text { of classical optimisation; } \\
\text { proving convexity can be } \\
\text { very challenging; } \\
\text { obtaining solutions can } \\
\text { be rather computationally } \\
\text { complex and time } \\
\text { consuming. }\end{array}$ \\
\hline 2. & $\begin{array}{l}\text { Studying problem } \\
\text { structure }\end{array}$ & $\begin{array}{l}\text { Decomposition; } \\
\text { linearisation; } \\
\text { relaxation; approximation; } \\
\text { reformulation. }\end{array}$ & $\begin{array}{l}\text { Solutions can be optimal } \\
\text { or very close to optimal; } \\
\text { computational complexity } \\
\text { is significantly lowered. }\end{array}$ & $\begin{array}{l}\text { Special features might be } \\
\text { unavailable or difficult to } \\
\text { find; transformed problem } \\
\text { may be a far cry from } \\
\text { the original; new problem } \\
\text { may generate more } \\
\text { decision variables than in } \\
\text { the original one; solutions } \\
\text { are mostly suboptimal. }\end{array}$ \\
\hline 3. & Heuristics & $\begin{array}{l}\text { Greedy algorithms; } \\
\text { water-filling algorithms; } \\
\text { pre-assignment and } \\
\text { reassignment } \\
\text { algorithms; iterative-based } \\
\text { and recursive-based } \\
\text { algorithms. }\end{array}$ & $\begin{array}{l}\text { Solutions are quick to find; } \\
\text { less computational } \\
\text { complexity; requires little } \\
\text { or no numerical analysis; } \\
\text { solutions usually } \\
\text { suboptimal but could be } \\
\text { close to optimal; approach } \\
\text { is suitable for large and } \\
\text { practical networks. }\end{array}$ & $\begin{array}{l}\text { Solutions are } \\
\text { problem-specific and } \\
\text { most times are not } \\
\text { transferable; solutions can } \\
\text { not be numerically } \\
\text { analysed; solutions are } \\
\text { always suboptimal. }\end{array}$ \\
\hline 4. & Meta-heuristics & $\begin{array}{l}\text { Genetic algorithms; } \\
\text { simulated annealing; } \\
\text { evolutionary algorithms; } \\
\text { tabu searches. }\end{array}$ & $\begin{array}{l}\text { Algorithms are mostly } \\
\text { nature-inspired; they make } \\
\text { use of stochastic } \\
\text { components (e.g. random } \\
\text { variables); they are good } \\
\text { with large, practical and/or } \\
\text { computationally } \\
\text { demanding problems that } \\
\text { have large search spaces; } \\
\text { they use 'tricks' so as to not } \\
\text { get stuck at a local optimal } \\
\text { but to try obtain a global } \\
\text { optimal solution. }\end{array}$ & $\begin{array}{l}\text { Solutions are not } \\
\text { transferable; solutions } \\
\text { cannot be analysed } \\
\text { numerically. }\end{array}$ \\
\hline 5. & $\begin{array}{l}\text { Multi-objective } \\
\text { optimisation (using game } \\
\text { theory) }\end{array}$ & $\begin{array}{l}\text { Cooperative game; } \\
\text { non-cooperative game; } \\
\text { Nash bargaining (Pareto } \\
\text { optimisation); Stackelberg } \\
\text { game. }\end{array}$ & $\begin{array}{l}\text { They are good with } \\
\text { problems that have } \\
\text { multiple objectives; they } \\
\text { employ ideas from game } \\
\text { theory to solve } \\
\text { optimisation problems; } \\
\text { they are useful for large, } \\
\text { practical networks with } \\
\text { large search spaces. }\end{array}$ & $\begin{array}{l}\text { Solution models can be } \\
\text { complex; they are not } \\
\text { transferable; there may be } \\
\text { difficulty in achieving } \\
\text { analytical modelling of } \\
\text { solutions. }\end{array}$ \\
\hline 6. & $\begin{array}{l}\text { Soft computing-based } \\
\text { optimisation }\end{array}$ & $\begin{array}{l}\text { Artificial intelligence; } \\
\text { neural networks; } \\
\text { Q-learning; fuzzy systems } \\
\text { etc. }\end{array}$ & $\begin{array}{l}\text { Software/computer-based } \\
\text { programming } \\
\text { are used in allocating } \\
\text { resources to users within } \\
\text { the network; the } \\
\text { developed programmes } \\
\text { use intelligent and very } \\
\text { powerful/sophisticated } \\
\text { techniques. }\end{array}$ & $\begin{array}{l}\text { They are very difficult and } \\
\text { complex to develop, } \\
\text { analyse and apply in real } \\
\text { life scenarios. }\end{array}$ \\
\hline
\end{tabular}

- Sequel to the point raised above, there is therefore no general or one-fits-all solution model or approach for all RA problems in CRN that has been established.
- It is observed that most RA models have neglected some important considerations and/or limiting factors of CRN that should have made the problem 
more realistic and close to practicality. For instance, the issue of heterogeneity in CRN, which would have created more practical scenarios, has been largely ignored by most authors in their RA problem development and solution investigations.

The reasons that can be construed for the issues raised above are the following realities still currently associated with CRN:

- There is a general difficulty in establishing, explaining and capturing all the details of CRN in one single model. As a result of the numerous and divergent architectures that have been postulated for CRN (as earlier explained), it would be very tedious, almost impossible, to develop RA models that will capture all of the important details in one spell or shot. Several small models that address specific areas of interest, while making assumptions on other details, are thus (or seems to be) the only currently meaningful approach to developing useful research models on the subject matter.

- There are no well-established standards in place yet for CRN, as it is still generally a work-in-progress. Though there have been attempts at defining and describing some form of standards (such as the IEEE 802.22 working group, which was set up to develop a standard for wireless regional area networks (WRAN) that would make use of, on a non-interfering basis, TV white spaces [89]), the fact remains that no standard has been fully established and accepted by all stakeholders for CRN to operate by.

- Optimisation, the main tool used in solving RA problems in CRN is, in itself, a diverse and dynamic problem-solving tool with multiple dimensions of interpretation and application for obtaining solutions to problems. Hence, arriving at a single generalised solution model for solving RA problems using optimisation is not very likely.

From the exposures and explanations thus far presented on RA in CRN, some open-ended problems that could potentially limit the productivity of CRN in its RA models, and therefore still require further investigations, have been identified. In this section, the most important problems are mentioned and discussed briefly. The openended problems, which could limit RA optimisation in $\mathrm{CRN}$, and suggested ideas for solutions, are discussed:

- Network heterogeneity: In all probability, CRN would almost certainly be a type of heterogeneous network (HetNet) or, at the least, it would bear certain semblance or cut across HetNet in some way. Therefore, proper classification and study of HetNet, and how it applies to CRN, would give the needed ideas on how to address the heterogeneity problem in CRN. Inclusion of heterogeneous classifications into the RA problems of CRN and studying it as such would therefore be a step in the right direction, as this would most likely bring the models closer to practicality.

- Limitation due to the level of permissible interference to PUs: In almost all the works on RA in CRN studied, the most prominent denominator, cutting across all kinds of architecture and RA problem definition, is the fact that the interference to PUs is a limiting constraint, probably the most limiting. The effects of this limiting constraint seem to be what hamper the progress and possibilities of CRN the most. Unfortunately, almost all of the works reviewed have only mentioned this problem, and of course, the authors have included it as one of the constraints in the optimisation problem but not much has been done towards mitigating its effect on the overall productivity of CRN. If CRN would ever achieve its ends, the problem of limitation due to interference to PUs must be adequately addressed. As a suggestion going forward, cooperative diversity, not just applied for spectrum sensing but for RA, if properly investigated and employed, could be a promising solution to the interference limitations in RA for CRN.

- Data buffering in CRN: The possibility of delay in data transmission has seldom been factored into the RA problems of CRN. Almost all works reviewed have equally neglected this concept in their problem definition. In reality, for heterogeneous CRN particularly, delay tolerance of different users might differ significantly, and there might be need for queue considerations. To analyse RA models that capture such possibilities, the use of queueing theory could help in addressing the delay issues. Hence, RA problems in CRN that factor this into their designs, especially when heterogeneous considerations are also involved, would be a good research focus.

\section{Conclusions}

In this paper, a critical review of the major approaches to RA in CRN, as employed by most researchers in the field, has been provided. The review identifies what the important challenges with RA in wireless communication are and the specific peculiarities of CRN that render such problems even more exacerbating, thus making it very difficult to investigate solutions. Thereafter, the paper reveals the various ideas, methods and reasoning that have been employed by researchers in seeking viable solutions to the RA problems in CRN. From the review, open-ended problems that could limit RA solutions in CRN are identified and 
some ideas for possible investigation are then postulated. The ideas put forth can indeed form a good basis for further investigations in the field of CRN, especially as regards devising solution models for addressing its RA problems.

\section{Acknowledgements}

This work was supported by the SENTECH Chair in Broadband Wireless Multimedia Communications (BWMC), a telecommunications research group in the Department of Electrical, Electronics and Computer Engineering at the University of Pretoria, South Africa.

\section{Competing interests}

The authors declare that they have no competing interests.

\section{Author details}

${ }^{1}$ Department of Electrical, Electronic and Computer Engineering, University of Pretoria, Pretoria, South Africa. ${ }^{2}$ Department of Electrical and Computer Engineering, University of Manitoba, Winnipeg, Canada.

Received: 9 February 2016 Accepted: 11 July 2016

Published online: 29 July 2016

\section{References}

1. BA Fette, Cognitive Radio Technology. Communications Engineering Series. (Newness (Elsevier) Publications, Burlington, 2006)

2. LE Doyle, Essentials of Cognitive Radio. The Cambridge Wireless Essentials Series. (Cambridge University Press, New York, 2009)

3. B Wang, KJR Liu, Advances in cognitive radio networks: a survey. IEEE J. Sel. Top. Signal Proc. 5(1), 5-23 (2011). doi:10.1109/JSTSP.2010.2093210

4. Y-C Liang, K-C Chen, GY Li, P Mahonen, Cognitive radio networking and communications: an overview. IEEE Trans. Veh. Technol. 60(7), 3386-3407 (2011). doi:10.1109/TVT.2011.2158673

5. X Liu, Y Zhang, Y Li, Z Zhang, K Long, in Communications and Networking in China (CHINACOM), 2013 8th International ICST Conference On. A survey of cognitive radio technologies and their optimization approaches, (2013), pp. 973-978. doi:10.1109/ChinaCom.2013.6694736

6. B Fette, in Military Communications Conference, MILCOM 2013 - 2013 IEEE. Fourteen years of cognitive radio development, (2013), pp. 1166-1175. doi:10.1109/MILCOM.2013.200

7. K-C Chen, Y-J Peng, N Prasad, Y-C Liang, S Sun, in Proceedings of the 2Nd International Conference on Ubiquitous Information Management and Communication. ICUIMC '08. Cognitive radio network architecture: Part ii - trusted network layer structure (ACM, New York, 2008), pp. 120-124. doi:10.1145/1352793.1352818. http://doi.acm.org/10.1145/1352793. 1352818

8. CXin, X Cao, in Global Telecommunications Conference, 2009. GLOBECOM 2009. IEEE. A cognitive radio network architecture without control channel, (2009), pp. 1-6. doi:10.1109/GLOCOM.2009.5426138

9. D Xu, Q Zhang, Y Liu, Y Xu, P Zhang, in Vehicular Technology Conference (VTC Fall), 2012 IEEE. An architecture for cognitive radio networks with cognition, self-organization and reconfiguration capabilities, (2012), pp. 1-5. doi:10.1109/NTCFall.2012.6398986

10. A Amanna, JH Reed, in IEEE SoutheastCon 2010 (SoutheastCon), Proceedings of The. Survey of cognitive radio architectures, (2010), pp. 292-297. doi:10.1109/SECON.2010.5453869

11. M Monemi, M Rasti, E Hossain, in Communications (ICC), 2015 IEEE International Conference On. Characterizing feasible interference region for underlay cognitive radio networks, (2015), pp. 7603-7608. doi:10.1109/ICC.2015.7249542

12. W Guo, X Huang, in Military Communications Conference, 2009. MILCOM 2009. IEEE. Maximizing throughput for overlaid cognitive radio networks, (2009), pp. 1-7. doi:10.1109/MILCOM.2009.5380005

13. W-L Chin, J-M Lee, Spectrum sensing scheme for overlay cognitive radio networks. Electron. Lett. 51(19), 1552-1554 (2015). doi:10.1049/el.2015.1112

14. S Senthuran, A Anpalagan, O Das, Throughput analysis of opportunistic access strategies in hybrid underlay overlay cognitive radio networks. IEEE
Trans. Wirel. Commun. 11(6), 2024-2035 (2012). doi:10.1109/TWC.2012.032712.101209

15. J Lai, E Dutkiewicz, RP Liu, R Vesilo, in Global Communications Conference (GLOBECOM), 2012 IEEE. Comparison of cooperative spectrum sensing strategies in distributed cognitive radio networks, (2012), pp. 1513-1518. doi:10.1109/GLOCOM.2012.6503328

16. M Nabil, W El-Sayed, M Elnainay, in Wireless Communications and Mobile Computing Conference (IWCMC), 2014 International. A cooperative spectrum sensing scheme based on task assignment algorithm for cognitive radio networks, (2014), pp. 151-156. doi:10.1109/IWCMC.2014.6906348

17. $H X u, B L i$, in INFOCOM, 2010 Proceedings IEEE. Efficient resource allocation with flexible channel cooperation in OFDMA cognitive radio networks, (2010), pp. 1-9. doi:10.1109/INFCOM.2010.5462169

18. $\mathrm{HXu}, \mathrm{B} \mathrm{Li}$, Resource allocation with flexible channel cooperation in cognitive radio networks. IEEE Trans. Mob. Comput. 12(5), 957-970 (2013). doi:10.1109/TMC.2012.62

19. Z Mao, XWang, Efficient optimal and suboptimal radio resource allocation in ofdma system. IEEE Trans. Wirel. Commun. 7(2), 440-445 (2008). doi:10.1109/TWC.2008.060546

20. C Turgu, C Toker, in Statistical Signal Processing, 2009. SSP '09. IEEE/SP 15th Workshop On. A low complexity resource allocation algorithm for OFDMA systems, (2009), pp. 689-692. doi:10.1109/SSP.2009.5278481

21. C Shi, Y Wang, P Zhang, in Global Communications Conference (GLOBECOM), 2012 IEEE. Joint spectrum sensing and resource allocation for multi-band cognitive radio systems with heterogeneous services, (2012), pp. 1180-1185. doi:10.1109/GLOCOM.2012.6503273

22. $\mathrm{X}$ Yu, T LV, P Chang, Y Li, in Wireless Communications Networking and Mobile Computing (WiCOM), 20106 th International Conference On. Enhanced efficient optimal and suboptimal radio resource allocation in ofdma system, (2010), pp. 1-4. doi:10.1109/WICOM.2010.5600209

23. S Kim, BG Lee, D Park, Energy-per-bit minimized radio resource allocation in heterogeneous networks. IEEE Trans. Wirel. Commun. 13(4), 1862-1873 (2014). doi:10.1109/TWC.2014.022114.130443

24. S Bashar, Z Ding, Admission control and resource allocation in a heterogeneous OFDMA wireless network. IEEE Trans. Wirel. Commun. 8(8), 4200-4210 (2009). doi:10.1109/TWC.2009.080844

25. TVilla, R Merz, R Knopp, in Global Communications Conference (GLOBECOM), 2013 IEEE. Dynamic resource allocation in heterogeneous networks, (2013), pp. 1915-1920. doi:10.1109/GLOCOM.2013.6831354

26. EB Rodrigues, F Casadevall, in European Wireless, 2012. EW. 18th European Wireless Conference. Rate adaptive resource allocation with fairness control for ofdma networks, (2012), pp. 1-8

27. M Fang, G Song, in ICT and Energy Efficiency and Workshop on Information Theory and Security (CIICT 2012), Symposium On. Adaptive resource allocation schemes for OFDMA systems with proportional rate constraint, (2012), pp. 106-110. doi:10.1049/cp.2012.1872

28. SCicalo, V Tralli, Adaptive resource allocation with proportional rate constraints for uplink SC-FDMA systems. IEEE Commun. Lett. 18(8), 1419-1422 (2014). doi:10.1109/LCOMM.2014.2332997

29. H Liming, X Lin, in EUROCON 2009, EUROCON '09. IEEE. Margin adaptive resource allocation with long-term rate fairness considered in downlink OFDMA systems, (2009), pp. 1919-1923. doi:10.1109/EURCON.2009.5167909

30. MH Hassan, MJ Hossain, Cooperative beamforming for cognitive radio systems with asynchronous interference to primary user. IEEE Trans. Wirel. Commun. 12(11), 5468-5479 (2013). doi:10.1109/TWC.2013.092013.121275

31. M Pischella, J-C Belfiore, Distributed margin adaptive resource allocation in MIMO OFDMA networks. IEEE Trans. Commun. 58(8), 2371-2380 (2010). doi:10.1109/TCOMM.2010.08.080678

32. BS Awoyemi, BT Maharaj, AS Alfa, in AFRICON, 2015 IEEE. Qos provisioning in heterogeneous cognitive radio networks through dynamic resource allocation, (2015)

33. J-C Liang, J-C Chen, Resource allocation in cognitive radio relay networks. IEEE J. Sel. Areas Commun. 31(3), 476-488 (2013). doi:10.1109/JSAC.2013.130313

34. Y Tachwali, F Basma, HH Refai, Cognitive radio architecture for rapidly deployable heterogeneous wireless networks. IEEE Trans. Consum. Electron. 56(3), 1426-1432 (2010). doi:10.1109/TCE.2010.5606279 
35. WL Winston, M Venkataramanan, Introduction to Mathematical Programming, 4th edn. (Thompson Brooks/Cole, Pacific Grove, 2003)

36. P Pedregal, Introduction to Optimization. Texts in Applied Mathematics. (Springer, New York, 2004)

37. KP Edwin, HZ Stanislaw, An Introduction to Optimization. (Wiley, West Sussex UK, 2013)

38. SP Boyd, L Vandenberghe, Convex Optimization. Berichte über verteilte messysteme. (Cambridge University Press, 2004). https://books.google.co. za/books?id=mYm0bLd3fcoC

39. RW Floyd, Nondeterministic algorithms. J. ACM. 14(4), 636-644 (1967). doi:10.1145/321420.321422

40. O Tripp, E Koskinen, M Sagiv, Turning nondeterminism into parallelism. SIGPLAN Not. 48(10), 589-604 (2013). doi:10.1145/2544173.2509533

41. G Zhao, J Li, K Lee, JB Song, Optimal frequency-time allocation in cognitive radio wireless mesh networks. IETE Tech. Rev. 28(5), 434-444 (2011). doi:10.4103/0256-4602.85976. http://www.tandfonline.com/doi/ pdf/10.4103/0256-4602.85976

42. A El Shafie, A Sultan, T Khattab, in Wireless Communications and Networking Conference (WCNC), 2014 IEEE. Band allocation for cognitive radios with buffered primary and secondary users, (2014), pp. 1508-1513. doi:10.1109/WCNC.2014.6952433

43. F Wang, W Wang, in Global Telecommunications Conference (GLOBECOM 2010), 2010 IEEE. Robust beamforming and power control for multiuser cognitive radio network, (2010), pp. 1-5. doi:10.1109/GLOCOM.2010.5683735

44. Z-Q Luo, W Yu, An introduction to convex optimization for communications and signal processing. IEEE J. Sel. Areas Commun. 24(8), 1426-1438 (2006). doi:10.1109/JSAC.2006.879347

45. Y Rahulamathavan, K Cumanan, S Lambotharan, in Signal Processing Advances in Wireless Communications (SPAWC), 2010 IEEE Eleventh International Workshop On. Optimal resource allocation techniques for MIMO-OFDMA based cognitive radio networks using integer linear programming, (2010), pp. 1-5. doi:10.1109/SPAWC.2010.5670885

46. M-S Cheon, S Ahmed, F Al-Khayyal, A branch-reduce-cut algorithm for the global optimization of probabilistically constrained linear programs. Math. Program. 108(2), 617-634 (2006). doi:10.1007/s10107-006-0725-5

47. M Bocus, J Coon, C Canagarajah, J McGeehan, S Armour, A Doufexi, Resource allocation for OFDMA-based cognitive radio networks with application to h.264 scalable video transmission. EURASIP J. Wirel. Commun. Netw. 2011 (1), 245673 (2011). doi:10.1155/2011/245673

48. L Wang, W Xu, Z He, J Lin, in Power Electronics and Intelligent Transportation System (PEITS), 2009 2nd International Conference On. Algorithms for optimal resource allocation in heterogeneous cognitive radio networks, vol. 2, (2009), pp. 396-400. doi:10.1109/PEITS.2009.5406755

49. R Xie, FR Yu, H Ji, Dynamic resource allocation for heterogeneous services in cognitive radio networks with imperfect channel sensing. IEEE Trans. Veh. Technol. 61(2), 770-780 (2012). doi:10.1109/TVT.2011.2181966

50. S Wang, M Ge, C Wang, Efficient resource allocation for cognitive radio networks with cooperative relays. IEEE J. Sel. Areas Commun. 31(11), 2432-2441 (2013). doi:10.1109/JSAC.2013.131128

51. S Du, F Huang, S Wang, Power allocation for orthogonal frequency division multiplexing-based cognitive radio networks with cooperative relays. IET Commun. 8(6), 921-929 (2014). doi:10.1049/iet-com.2013.0379

52. W-C Pao, Y-F Chen, Adaptive gradient-based methods for adaptive power allocation in OFDM-based cognitive radio networks. IEEE Trans. Veh. Technol. 63(2), 836-848 (2014). doi:10.1109/TVT.2013.2272804

53. J Zhang, Z Zhang, H Luo, A Huang, in Global Telecommunications Conference, 2008. IEEE GLOBECOM 2008. IEEE. A column generation approach for spectrum allocation in cognitive wireless mesh network, (2008), pp. 1-5. doi:10.1109/GLOCOM.2008.ECP.593

54. Z He, S Mao, S Kompella, A decomposition approach to quality-driven multiuser video streaming in cellular cognitive radio networks. IEEE Trans. Wirel. Commun. 15(1), 728-739 (2016). doi:10.1109/TWC.2015.2477509

55. MG Adian, H Aghaeinia, Optimal resource allocation for opportunistic spectrum access in multiple-input multiple-output-orthogonal frequency division multiplexing based cooperative cognitive radio networks. IET Signal Process. 7(7), 549-557 (2013). doi:10.1049/iet-spr.2012.0401

56. L Zheng, CW Tan, Cognitive radio network duality and algorithms for utility maximization. IEEE J. Sel. Areas Commun. 31(3), 500-513 (2013) doi:10.1109/JSAC.2013.130315
57. Y Shi, YT Hou, in INFOCOM 2008. The 27th Conference on Computer Communications. IEEE. A distributed optimization algorithm for multi-hop Cognitive radio networks, (2008). doi:10.1109/INFOCOM.2008.186

58. P Li, S Guo, W Zhuang, B Ye, On efficient resource allocation for cognitive and cooperative communications. IEEE J. Sel. Areas Commun. 32(2), 264-273 (2014). doi:10.1109/JSAC.2014.141207

59. F Chen, W Xu, Y Guo, J Lin, M Chen, in Communications and Networking in China (CHINACOM), 2013 8th International ICST Conference On. Resource allocation in OFDM-based heterogeneous cognitive radio networks with imperfect spectrum sensing and guaranteed QOS, (2013), pp. 46-51. doi:10.1109/ChinaCom.2013.6694563

60. M Hasegawa, H Hirai, K Nagano, H Harada, K Aihara, Optimization for centralized and decentralized cognitive radio networks. Proc. IEEE. 102(4), 574-584 (2014). doi:10.1109/JPROC.2014.2306255

61. Y Rahulamathavan, S Lambotharan, C Toker, A Gershman, Suboptima recursive optimisation framework for adaptive resource allocation in spectrum-sharing networks. IET Signal Process. 6(1), 27-33 (2012). doi:10.1049/iet-spr.2011.0005

62. Y Rahulamathavan, K Cumanan, L Musavian, S Lambotharan, in Statistical Signal Processing, 2009. SSP'09. IEEE/SP 15th Workshop On. Optimal subcarrier and bit allocation techniques for cognitive radio networks using integer linear programming, (2009), pp. 293-296. doi:10.1109/SSP.2009.5278582

63. Y Rahulamathavan, K Cumanan, R Krishna, S Lambotharan, in Cognitive Wireless Systems (UKIWCWS), 2009 First UK-India International Workshop On. Adaptive subcarrier and bit allocation techniques for MIMO-OFDMA based uplink cognitive radio networks, (2009), pp. 1-5. doi:10.1109/UKIWCWS.2009.5749387

64. P Mitran, LB Le, C Rosenberg, Queue-aware resource allocation for downlink OFDMA cognitive radio networks. IEEE Trans. Wirel. Commun. 9(10), 3100-3111 (2010). doi:10.1109/TWC.2010.080610.090901

65. E Driouch, W Ajib, A Ben Dhaou, in Computing, Networking and Communications (ICNC), 2012 International Conference On. A greedy spectrum sharing algorithm for cognitive radio networks, (2012), pp. 1010-1014. doi:10.1109/ICCNC.2012.6167359

66. T Peng, W Wang, Q Lu, W Wang, in Wireless Communications, Networking and Mobile Computing, 2007. WiCom 2007. International Conference On. Subcarrier allocation based on water-filling level in ofdma-based cognitive radio networks, (2007), pp. 196-199. doi:10.1109/WICOM.2007.56

67. A Zafar, M-S Alouini, Y Chen, RM Radaydeh, in Communications (ICC), 2012 IEEE International Conference On. New resource allocation scheme for cognitive relay networks with opportunistic access, (2012), pp. 5603-5607. doi:10.1109/ICC.2012.6364910

68. Y Liu, L Liu, C Xu, in Electric Information and Control Engineering (ICEICE), 2011 International Conference On. Spectrum underlay-based water-filling algorithm in cognitive radio networks, (2011), pp. 2614-2617. doi:10.1109/ICEICE.2011.5778318

69. RL Ujjwal, CS Rai, N Prakash, in Signal Processing and Integrated Networks (SPIN), 2014 International Conference On. Fair adaptive resource allocation algorithm for heterogeneous users in OFDMA system, (2014), pp. 402-406. doi:10.1109/SPIN.2014.6776987

70. MG Adian, H Aghaeinia, Y Norouzi, Optimal resource allocation for opportunistic spectrum access in heterogeneous MIMO cognitive radio networks. Trans. Emerg. Telecommun. Technol (2014). doi:10.1002/ett.2796

71. MG Adian, H Aghaeinia, Optimal resource allocation in heterogeneous MIMO cognitive radio networks. Wirel. Pers. Commun. 76(1), 23-39 (2014). doi:10.1007/s11277-013-1486-0

72. A Alshamrani, X Shen, L-L Xie, Qos provisioning for heterogeneous services in cooperative cognitive radio networks. IEEE J. Sel. Areas Commun. 29(4), 819-830 (2011). doi:10.1109/JSAC.2011.110413

73. I Boussaid, J Lepagnot, P Siarry, A survey on optimization metaheuristics. Inf. Sci. 237, 82-117 (2013). doi:10.1016/j.ins.2013.02.041.Prediction, Control and Diagnosis using Advanced Neural Computations

74. Y El Morabit, F Mrabti, EH Abarkan, in RFID And Adaptive Wireless Sensor Networks (RAWSN), 2015 Third International Workshop On. Spectrum allocation using genetic algorithm in cognitive radio networks, (2015), pp. 90-93. doi:10.1109/RAWSN.2015.7173287

75. L Zhu, Y Xu, J Chen, Z Li, in Computational Intelligence Communication Technology (CICT), 2015 IEEE International Conference On. The design of 
scheduling algorithm for cognitive radio networks based on genetic algorithm, (2015), pp. 459-464. doi:10.1109/CICT.2015.131

76. E Meshkova, J Riihijarvi, A Achtzehn, P Mahonen, in Global Telecommunications Conference, 2009. GLOBECOM 2009. IEEE. Exploring simulated annealing and graphical models for optimization in cognitive wireless networks, (2009), pp. 1-8. doi:10.1109/GLOCOM.2009.5425349

77. B Ye, M Nekovee, A Pervez, M Ghavami, in Cognitive Radio Oriented Wireless Networks and Communications (CROWNCOM), 20127 th International ICST Conference On. Tv white space channel allocation with simulated annealing as meta algorithm, (2012), pp. 175-179

78. SMotiian, M Aghababaie, H Soltanian-Zadeh, in Broadband Network and Multimedia Technology (IC-BNMT), 2011 4th IEEE International Conference On. Particle swarm optimization (pso) of power allocation in cognitive radio systems with interference constraints, (2011), pp. 558-562. doi:10.1109/ICBNMT.2011.6155997

79. S Ashrafinia, U Pareek, M Naeem, DC Lee, in Communications (ICC), 2012 IEEE International Conference On. Binary artificial bee colony for cooperative relay communication in cognitive radio systems, (2012), pp. 1550-1554. doi:10.1109//CC.2012.6364087

80. V Jayaraj, JJ Amalraj, S Hemalatha, An analysis of genetic algorithm and tabu search algorithm for channel optimization in cognitive adhoc networks. Int. J. Comput. Sci. Mob. Comput. 3(7), 60-69 (2014)

81. R Meng, Y Ye, N-g Xie, in Intelligent Control and Automation (WCICA), 2010 8th World Congress On. Multi-objective optimization design methods based on game theory, (2010), pp. 2220-2227. doi:10.1109/WCICA.2010.5554307

82. DN Nguyen, M Krunz, in Global Communications Conference (GLOBECOM), 2013 IEEE. Heterogeneous spectrum sharing with rate demands in cognitive MIMO networks, (2013), pp. 3054-3059. doi:10.1109/GLOCOM.2013.6831540

83. R Xie, FR Yu, H Ji, in Communications (ICC), 2012 IEEE International Conference On. Spectrum sharing and resource allocation for energy-efficient heterogeneous cognitive radio networks with femtocells, (2012), pp. 1661-1665. doi:10.1109/ICC.2012.6364100

84. R Xie, FR Yu, H Ji, Y Li, Energy-efficient resource allocation for heterogeneous cognitive radio networks with femtocells. IEEE Trans. Wirel. Commun. 11(11), 3910-3920 (2012). doi:10.1109/TWC.2012.092112.111510

85. M Venkatesan, A Kulkarni, Soft computing based learning for cognitive radio. Int. J. Rec. Trends Eng. Technol. 10(1), 112 (2014)

86. E Shakshuki, M Younas, A Ahmed, G Amel, S Anis, M Abdellatif, Ant 2012 and mobiwis 2012 resource allocation for multi-user cognitive radio systems using multi-agent q-learning. Procedia Comput. Sci. 10, 46-53 (2012). doi:10.1016/j.procs.2012.06.010

87. Y Huang, J Wang, H Jiang, in Networks Security Wireless Communications and Trusted Computing (NSWCTC), 2010 Second International Conference On. Modeling of learning inference and decision-making engine in cognitive radio, vol. 2, (2010), pp. 258-261. doi:10.1109/NSWCTC.2010.195

88. GV Lakhekar, RG Roy, in Circuit, Power and Computing Technologies (ICCPCT), 2014 International Conference On. A fuzzy neural approach for dynamic spectrum allocation in cognitive radio networks, (2014), pp. 1455-1461. doi:10.1109/ICCPCT.2014.7054970

89. C-W Pyo, X Zhang, C Song, M-T Zhou, H Harada, in Wireless Personal Multimedia Communications (WPMC), 201215 th International Symposium On. A new standard activity in ieee 802.22 wireless regional area networks: Enhancement for broadband services and monitoring applications in tv whitespace, (2012), pp. 108-112

90. MG Adian, H Aghaeinia, Optimal and sub-optimal resource allocation in multiple-input multiple-output-orthogonal frequency division multiplexing-based multi-relay cooperative cognitive radio networks. IET Commun. 8(5), 646-657 (2014). doi:10.1049/iet-com.2013.0676

91. S Wang, Z-H Zhou, M Ge, CWang, Resource allocation for heterogeneous cognitive radio networks with imperfect spectrum sensing. IEEE J. Sel. Areas Commun. 31(3), 464-475 (2013). doi:10.1 109/JSAC.2013.130312

92. M Ge, S Wang, in 2014 IEEE ICC - Cognitive Radio and Networks Symposium. On the resource allocation for multi-relay cognitive radio systems, (2014), pp. 1591-1595. doi:10.1109/NETECF.2010.5594302

\section{Submit your manuscript to a SpringerOpen ${ }^{\circ}$ journal and benefit from:}

- Convenient online submission

Rigorous peer review

- Immediate publication on acceptance

- Open access: articles freely available online

- High visibility within the field

- Retaining the copyright to your article

Submit your next manuscript at $\gg$ springeropen.com 